\title{
FLUCTUACIÓN POBLACIONAL DE XYLEBORUS VOLVULUS (F.) (COLEOPTERA: CURCULIONIDAE), EN LOCALIDADES DE TABASCO, MÉXICO
}

\author{
ANdrés BAÑos JUÁREZ, ${ }^{1}$ MANuel PÉREZ de LA CRUZ, ${ }^{1}$ SAÚl SÁNCHEZ \\ SOTO $^{2}$ \& SILVIA CAPELLO GARCÍA ${ }^{1}$ \\ ${ }^{1}$ Universidad Juárez Autónoma de Tabasco, División Académica de Ciencias Biológicas, Código \\ Postal 86150, Carretera Villahermosa-Cárdenas km $0.5 \mathrm{~s} / \mathrm{n}$ entronque a Bosque de Saloya, \\ Villahermosa, Tabasco, México. \\ ${ }^{2}$ Colegio de Postgraduados, Campus Tabasco, Apartado postal 24 86500, H. Cárdenas Tabasco, \\ México. \\ Correspondencia: perezmandoc@hotmail.com
}

Baños Juárez, A., Pérez de la Cruz, M., Sánchez Soto, S. \& Capello García, S. 2012. Fluctuación poblacional de Xyleborus volvulus (F.) (Coleoptera: Curculionidae), en localidades de Tabasco, México. Acta Zoológica Mexicana (n. s.), 28(3): 540-549.

RESUMEN. El objetivo de este estudio es dar a conocer la fluctuación poblacional de Xyleborus volvulus en seis localidades de Tabasco, México. Los métodos de captura utilizados fueron las trampas de alcohol, trampa de luz y captura directa sobre sus plantas huésped. Se obtuvo como resultado un total de 4600 especímenes con los tres métodos de colecta, siendo las trampas de alcohol las que registraron el mayor número de organismos con 2 927, seguidos de las trampas de luz con 1595 y por último sobre sus plantas huésped con 78. La fluctuación de $X$. volvulus presentó dos picos poblacionales en todas las localidades estudiadas, uno al inicio (enero-abril) y otro hacia el final (septiembre, noviembre y diciembre) del periodo de estudio, coincidiendo en la mayoría de los casos con el periodo de lluvia de la zona. Además se determinó que esta especie se encuentra presente todo el año pero con poblaciones bajas.

Palabras clave: Insectos, barrenadores, cacao, ambrosiales.

Baños Juárez, A., Pérez de la Cruz, M., Sánchez Soto, S. \& Capello García, S. 2012. Population dynamics of Xyleborus volvulus (F.) (Coleoptera: Curculionidae) in localities of Tabasco, Mexico. Acta Zoológica Mexicana (n. s.), 28(3): 540-549.

ABSTRACT. The aim of this study is to show the population dynamics of Xyleborus volvulus in six localities of Tabasco, Mexico. The trapping methods used were alcohol traps, light traps and directly capture from their host plants. The total number of specimens collected was 4600 , using the three methods of collection. Most organisms were collected using alcohol traps 2927 , followed by light traps with 1595 and finally from their host plants with 78. The fluctuation of $X$. volvulus showed two population peaks in all the localities studied, one at the beginning (January-April) and another one towards the end (September, November and December) of study period, which, in most cases, coincides with those

Recibido: 19/01/2012; aceptado: 28/08/2012. 
periods of rain in the area. Also, it was determined that this species is present throughout the year but with low populations.

Key words: Insects, borers, cocoa, ambrosial.

\section{INTRODUCCIÓN}

Xyleborus volvulus es un insecto barrenador conocido como escarabajo de ambrosia debido a que se alimenta de hongos, tienen cierta división del trabajo; algunas hembras limpian las galerías y regulan el crecimiento de hongos asociados, otras sirven como guardias en los sitios de entrada de los túneles y otras más se encargan de incrementar el tamaño de las galerías, vive en grupos de hasta 500 individuos dentro de un sistema de galerías. Presenta varias generaciones por año y sobrepuestas (Cibrián et al. 1995). Las hembras penetran a la madera de los árboles por medio de túneles cilíndricos realizando galerías en diferentes planos del tronco atacado. Desde el inicio del ataque las hembras van liberando esporas de los hongos asociados que germinan dando paso al crecimiento del micelio dentro de la madera hasta alcanzar la fructificación en las paredes de los túneles. Las hembras ovipositan en las partes de las galerías, las larvas y adultos se alimentan de las estructuras reproductoras del hongo. Los machos no tienen alas funcionales pero copulan con las nuevas hembras que eventualmente pueden salir a infestar a nuevos huéspedes (Wood 1982, Cibrián et al. 1995). Estos insectos infestan árboles aparentemente sanos, debilitados o recién muertos y trocería húmeda de diferentes diámetros y tamaños. Su daño principal es reducir la calidad de la madera por las horadaciones que producen, así como promover el establecimiento de hongos manchadores de la madera. En las áreas tropicales de México es uno de los escarabajos de mayor importancia económica, debido a su amplia distribución y capacidad para dañar trocería y estar involucrado en la muerte de Erythrina americana que se usa como sombra en el cultivo de cacao (Cibrián et al. 1995). El estudio fue dirijido para conocer la fluctuación poblacional de $X$. volvulus en seis localidades de Tabasco, México y tener una idea del estado de las poblaciones en diferentes localidades y se implementaron diferentes métodos de muestreos con la finalidad de observar si éstos insectos pueden ser colectados a futuro de manera eficiente con algún método en particular y poder sugerirlos como métodos de monitoreo de las poblaciones de éstos insectos.

\section{MATERIALES Y MÉTODO}

El estudio se realizó en el Jardín Botánico José Narciso Rovirosa (JBU) de la División Académica de Ciencias Biológicas durante los meses de noviembre 2010 a octubre 2011, en la Reserva Ecológica de la Chontalpa (REC) durante febrero 2010 a enero 2011 y en cuatro plantaciones de cacao durante el año 2007 en Tabasco, México.

El Jardín Botánico José Narciso Rovirosa se encuentra establecido dentro de las instalaciones de la División Académica de Ciencias Biológicas, ubicado al oeste de 
la ciudad de Villahermosa en el $\mathrm{km} 0.5$ de la carretera 180 (costera del golfo, tramo Villahermosa-Cárdenas) en el municipio del Centro, Tabasco. Se localiza entre las coordenadas $17^{\circ} 59^{\prime} 26^{\prime \prime}$ y $17^{\circ} 59^{\prime} 17^{\prime \prime}$ latitud norte y $92^{\circ} 58^{\prime} 16^{\prime \prime}$ y $92^{\circ} 58^{\prime} 37^{\prime \prime}$ longitud oeste con una superficie de 10 ha con vegetación secundaria (acahual) y porción de selva baja espinosa perennifolia de tinto (Haematoxylum campechianum) (Ascencio \& Maldonado 2002).

La Reserva Ecológica de la Chontalpa se localiza en el municipio de Cárdenas, en el Kilómetro 21 de la carretera Cárdenas-Coatzacoalcos, Veracruz tiene una ex-

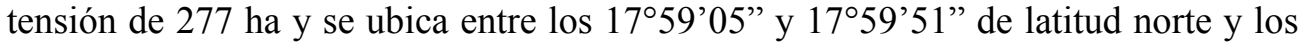
$93^{\circ} 34^{\prime} 13^{\prime \prime}$ y $93^{\circ} 35^{\prime} 33^{\prime \prime}$ de latitud oeste. La precipitación anual promedio es de 2256 $\mathrm{mm}$. El clima es denominado Am (f), cálido húmedo con lluvias en verano. Presenta una vegetación de bosques tropical perennifolio, que corresponde a selva mediana perennifolia. La especie dominante es Bravaisia integerrima conocida localmente como "conacoite" o "cintopie" (Palma-López et al. 1999).

En la primera plantación de cacao se utilizó un predio de seis hectáreas de 37 años de edad, la cual se localiza entre las coordenadas $17^{\circ} 59^{\prime} 23^{\prime \prime}$ de latitud norte y 93³7'09" de longitud oeste, en el Ejido Las Delicias del municipio de Teapa, Tabasco, México (Teapa). El clima es cálido húmedo con lluvias todo el año (Af). La precipitación alcanza una media anual de $3424 \mathrm{~mm}$ (INEGI 2006). La segunda plantación corresponde a una hectárea de aproximadamente 38 años de edad, localizada entre las coordenadas $17^{\circ} 37^{\prime} 19^{\prime \prime}$ de latitud norte y $92^{\circ} 27^{\prime} 56^{\prime \prime}$ de longitud oeste, en el kilómetro 21 de la carretera Cárdenas, Tabasco-Coatzacoalcos, Veracruz, México $(\mathrm{km} \mathrm{21})$. La tercera plantación consta de dos hectáreas de 10 años de edad, localizadas entre las coordenadas $17^{\circ} 58^{\prime} 09^{\prime \prime}$ de latitud norte y 9320'55" de longitud oeste en el Ejido El Bajío 2a sección (El Bajío). La cuarta plantación tuvo una extensión de 3.3 ha con 45 años de edad localizada entre las coordenadas $18^{\circ} 02^{\prime} 34^{\prime \prime}$ de latitud norte y $93^{\circ} 22^{\prime} 44^{\prime \prime}$ de longitud oeste en el Ejido Río Seco $2^{\mathrm{a}}$ sección (Río Seco). Las tres últimas plantaciones pertenecen a Cárdenas Tabasco y todas se encuentran establecidas en un marco real de $4 \times 4 \mathrm{~m}$. Su fisiografía corresponde a la llanura costera del Golfo Sur, con clima cálido húmedo con abundantes lluvias en verano (Am), la temperatura promedio es de $26{ }^{\circ} \mathrm{C}$. La precipitación media anual es de $1993 \mathrm{~mm}$ (INEGI 2005). Todas las localidades no sobrepasan los $20 \mathrm{msnm}$.

Captura de $X$. volvulus con trampas cebadas con alcohol etílico. Se instalaron cinco trampas de alcohol en una hectárea ubicada en el centro de cada localidad (excepto en el JBU que se instalaron dos trampas en las áreas de mayor vegetación), a una altura de $1.5 \mathrm{~m}$, a $50 \mathrm{~m}$ de distancia entre ellas formando un cuadrado con una trampa en el centro, utilizando como atrayente alcohol etílico de uso comercial al $70 \%$. La recolección de los insectos atraídos en cada una de las trampas se realizó quincenalmente, los especímenes se conservaron en alcohol al 70\% para su posterior determinación. En cada recolección se procedió a reponer el atrayente (Bustamante \& Atkinson 1984, Iturre \& Darchuck 1996, Pérez et al. 2009). 
Captura de $X$. volvulus con trampa de luz fluorescente. Se utilizó una trampa similar a la de alcohol para recolectar a los insectos sustituyendo el material atrayente por una fuente de luz fluorescente proporcionada por una lámpara recargable marca Lloyd's de 20 watts con una duración aproximada de 4 horas, colocada ésta a un costado de la trampa. La trampa se instaló en el centro de la plantación en cada una de las localidades de estudio (este método sólo fue usado en las plantaciones de cacao) a una altura de $1.5 \mathrm{~m}$ a las 18:00 horas y fue levantada al día siguiente; los muestreos se realizaron mensualmente. Los especímenes se colectaron en alcohol al 70\% para su posterior determinación (Pérez et al. 2009).

Captura directa de $X$. volvulus en sus plantas huésped. La recolecta consistió en hacer revisiones directamente sobre las partes de las plantas (tallo, ramas y frutos) en donde se observó la presencia de estos insectos y el número de plantas revisadas se determinó en base a la disponibilidad del material vegetal en las localidades estudiadas (Bustamante \& Atkinson 1984, Pérez et al. 2009). Los resultados se analizan bajo estadística descriptiva utilizando cuadros y figuras.

Identificación del material biológico. La determinación taxonómica del insecto se realizó mediante el uso de claves taxonómicas (Wood 1982, 1986) y comparaciones con material depositado en la colección del Colegio de Postgraduados Campus Montecillo, México (CEAM).

\section{RESULTADOS}

Se colectaron 4600 especímenes de $X$. volvulus con los tres métodos de colecta, siendo las trampas de alcohol las que registraron el mayor número de organismos con 2927, seguidos de las trampas de luz con 1595 y por último sobres sus plantas huésped con 78. En Teapa se registró el mayor número de insectos con 1304 y el menor lo presentó la REC con 198 (Cuadro 1).

Cuadro 1. Abundancia total de $X$. volvulus capturados con diferentes métodos de colecta en localidades de Tabasco, México.

\begin{tabular}{lcccc}
\hline \multicolumn{1}{c}{ Localidades } & Trampas de alcohol & Trampas de luz & Huésped & Total \\
\hline JBU & 1231 & N.E. & N.E. & 1231 \\
REC & 198 & N.E. & N.E. & 198 \\
Río Seco & 136 & 350 & 17 & 503 \\
km 21 & 516 & 479 & 2 & 997 \\
Teapa & 617 & 651 & 36 & 1304 \\
El Bajío & 229 & 115 & 23 & 367 \\
Total & $\mathbf{2 9 2 7}$ & $\mathbf{1 5 9 5}$ & $\mathbf{7 8}$ & 4600 \\
\hline
\end{tabular}

N.E. No Evaluado 
La fluctuación poblacional de $X$. volvulus capturado con trampas de alcohol en el JBU presentó dos picos poblacionales marcados uno durante enero y el otro en septiembre, capturándose individuos durante todo el periodo de estudio pero con poblaciones bajas la mayor parte del año (Fig. 1). Algo similar fué registrado en la

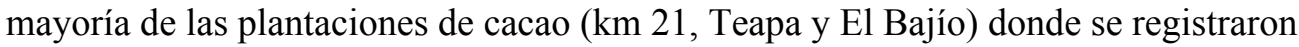
dos picos poblacionales marcados uno en marzo y otro al final del periodo de estudio (diciembre), siendo los meses de mayo a octubre donde se registraron las poblaciones con menor abundancia. En las plantaciones de cacao, Teapa y el km 21 registraron las poblaciones de insectos más elevadas destacándose con picos más altos en comparación con Río Seco y El Bajío (Fig. 2). En la REC $X$. volvulus registró un comportamiento poblacional similar a lo observado en las otras localidades estudiadas, con dos picos poblacionales marcados en abril y diciembre (Fig. 3). Cabe señalar que los picos poblacionales registraron una tendencia de presentarse hacia el inicio y el final del año de estudio en la mayoría de las localidades estudiadas, periodos considerados de lluvia y alto porcentaje de humedad (Figs. 4, 5 y 6).

La fluctuación poblacional de $X$. volvulus capturado en trampas de luz en las plantaciones de cacao (Río Seco, km 21, Teapa y El Bajío) presentó picos poblacionales marcados al inicio (febrero) y al final (noviembre) del año de estudio, coincidiendo con los resultados obtenidos en las trampas de alcohol en los mismos sitios (Fig. 7).

\section{DISCUSIÓN}

De manera general los máximos picos poblacionales registrados en la fluctuación po-

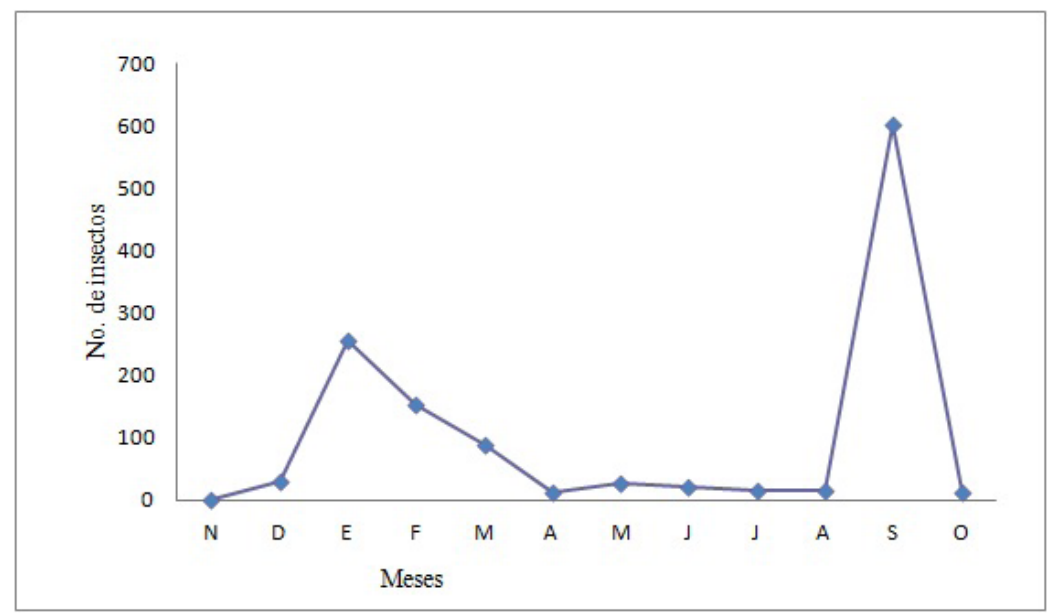

Figura 1. Fluctuación poblacional de $X$. volvulus capturados con trampas de alcohol en el Jardín Botánico José Narciso Rovirosa (JBU). 


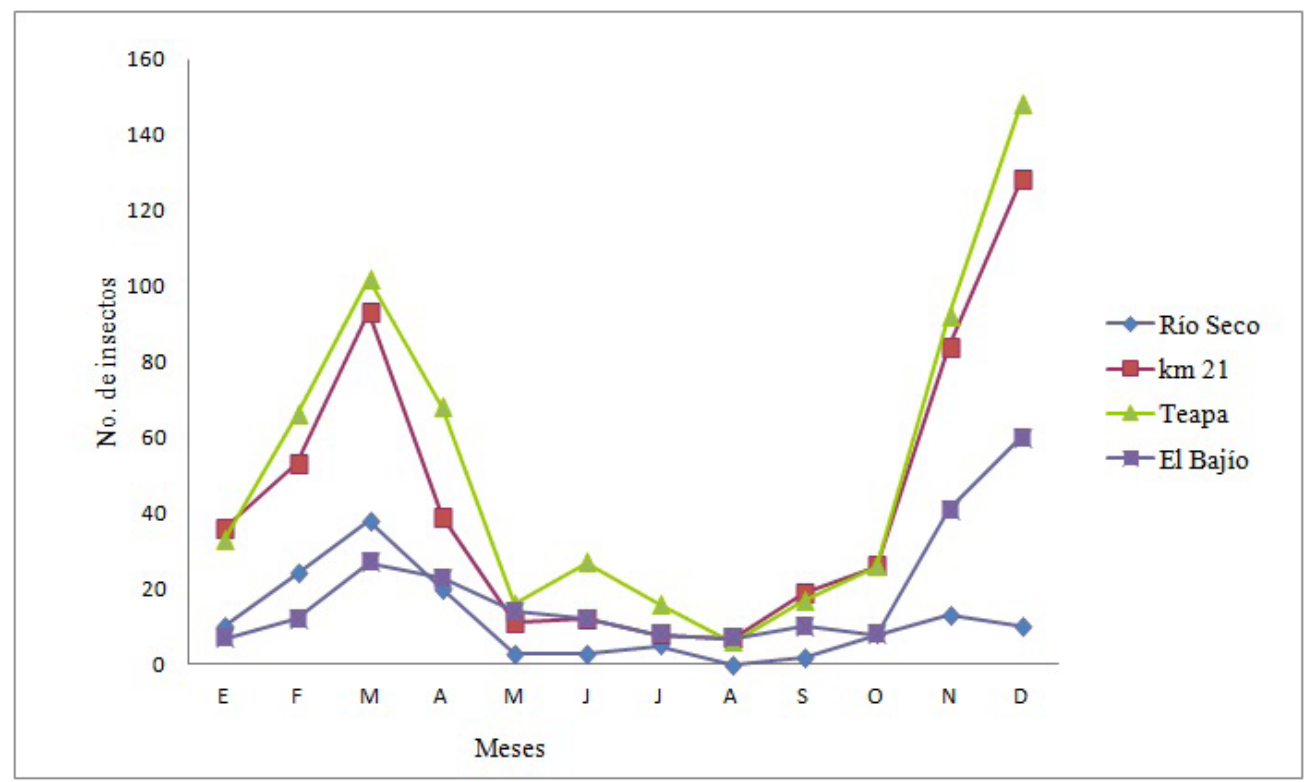

Figura 2. Fluctuación poblacional de $X$. volvulus capturado en trampas de alcohol en plantaciones de cacao en Tabasco, México.

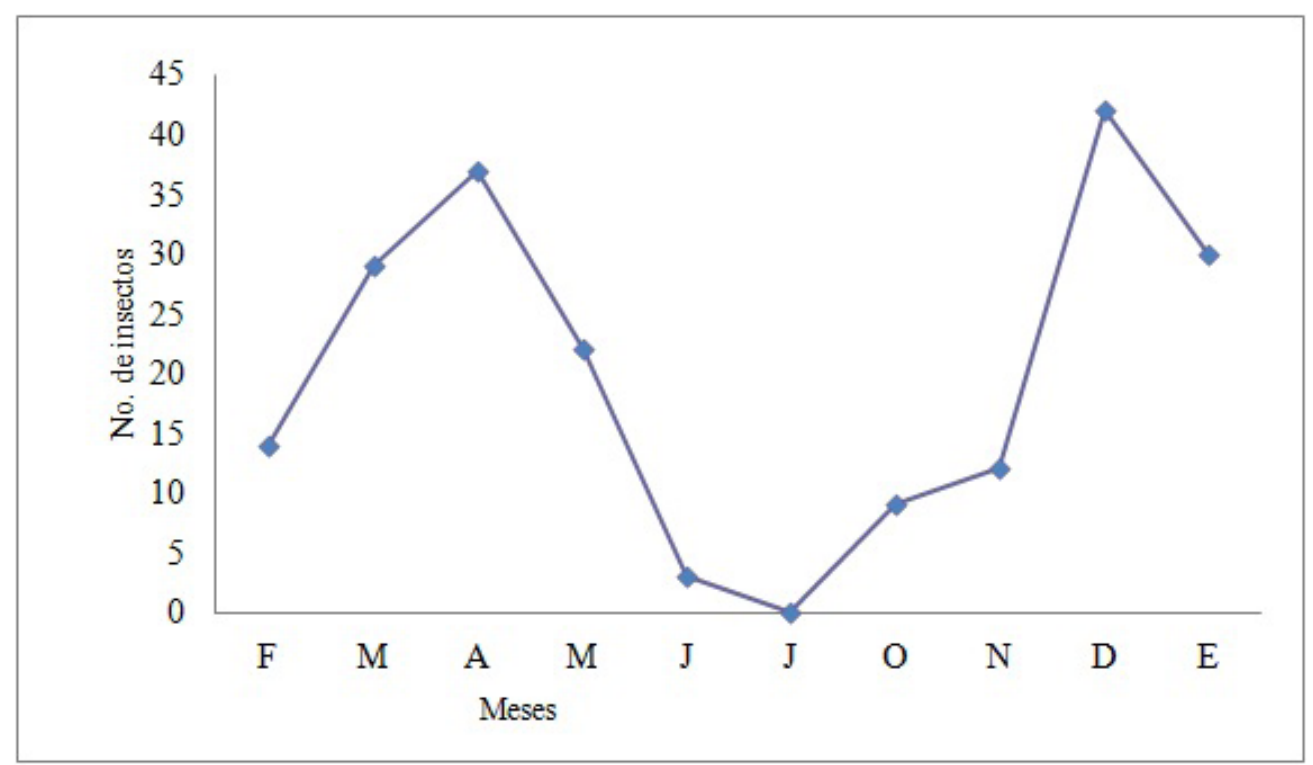

Figura 3. Fluctuación poblacional de $X$. volvulus capturado en trampas de alcohol en la Reserva Ecológica de la Chontalpa (REC) en Tabasco, México. 


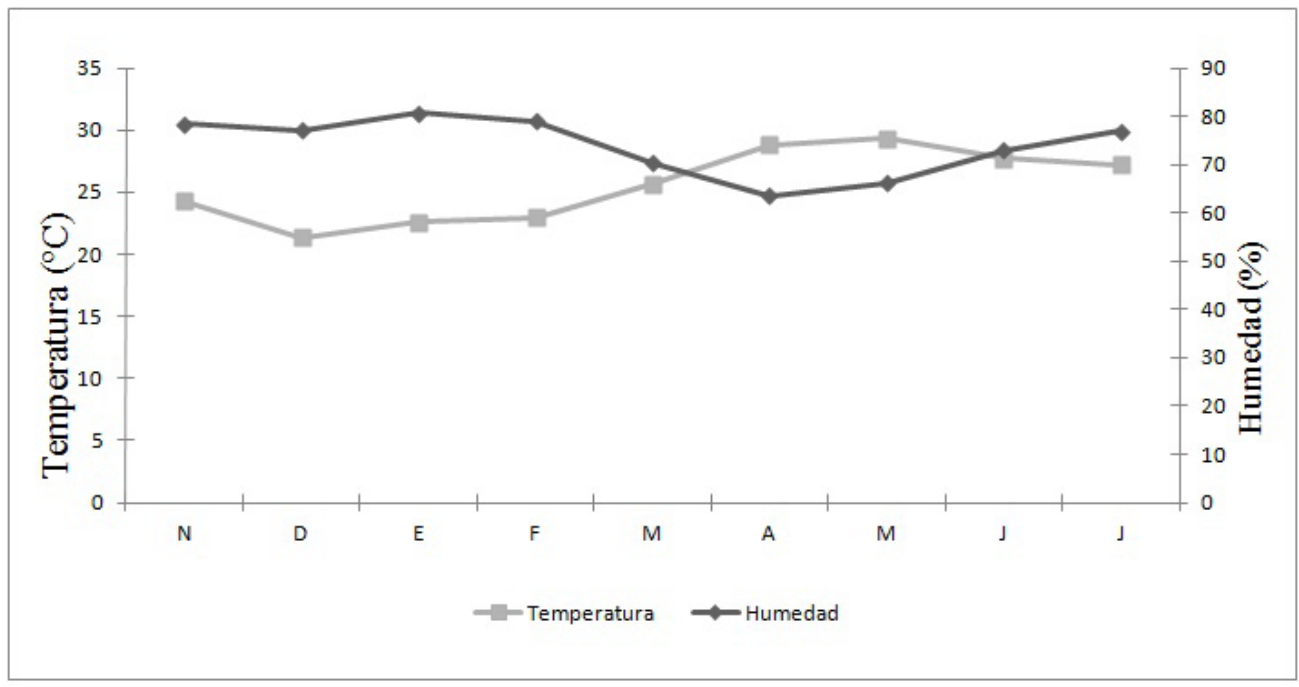

Figura 4. Promedios mensuales de temperatura $\left({ }^{\circ} \mathrm{C}\right)$ y humedad relativa $(\%)$ en JBU durante el periodo de estudio.

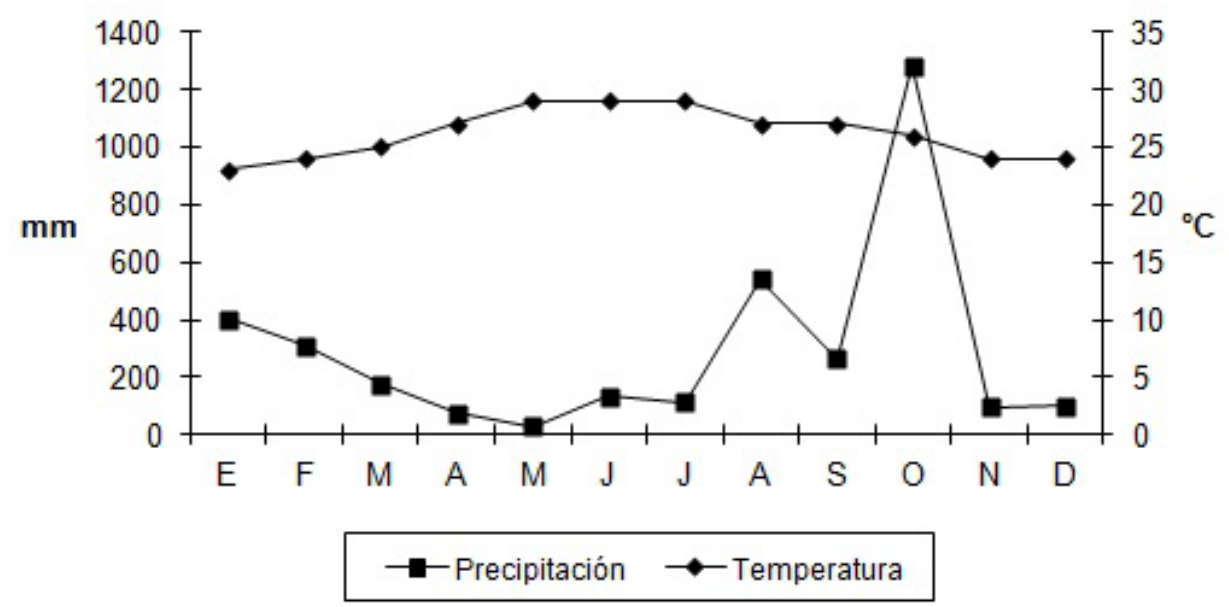

Figura 5. Promedios mensuales de precipitación $(\mathrm{mm})$ y temperatura $\left({ }^{\circ} \mathrm{C}\right)$ en el año 2007 en Teapa, Tabasco. 


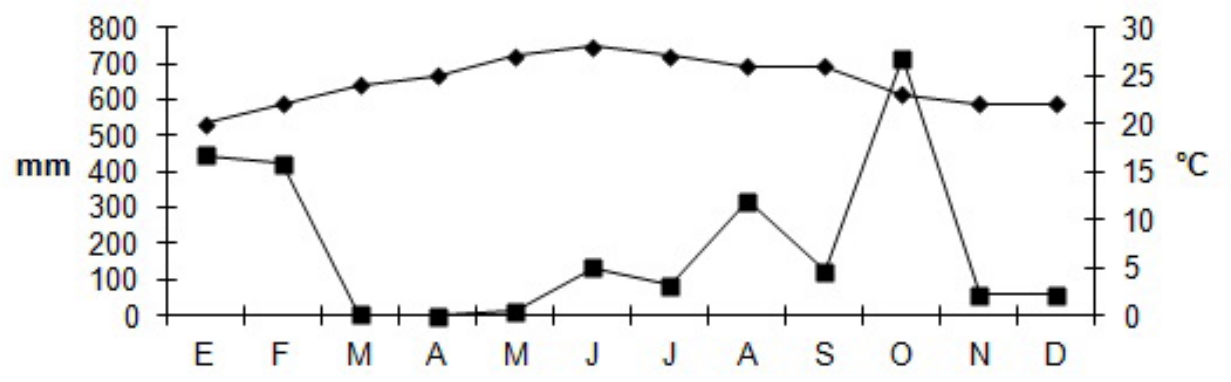

Figura 6. Promedios mensuales de precipitación $(\mathrm{mm})$ y temperatura $\left({ }^{\circ} \mathrm{C}\right)$ en el año 2007 en Cárdenas, Tabasco.

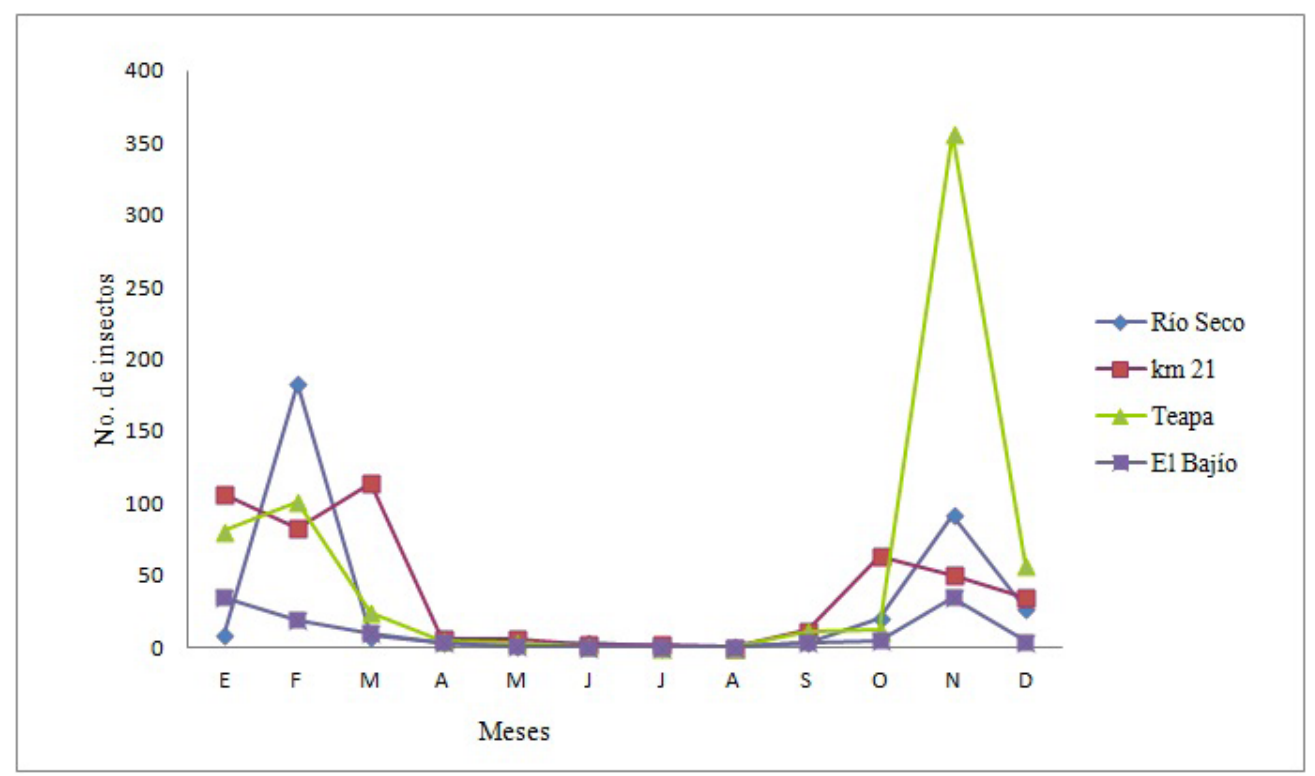

Figura 7. Fluctuación poblacional de $X$. volvulus capturado en trampas de luz en plantaciones de cacao en Tabasco, México. 
blacional de $X$. volvulus en la mayoría de las localidades difiere con los observado en $X$. paraguayensis, $X$. affinis en zonas reforestadas con Eucalyptus grandis en Minas Gerais, Brasil donde se han registrado en periodos secos (Morales et al. 2000); en este estudio tienden a presentarse dentro de periodos considerados lluviosos, excepto Abril en la Rec que es el mes junto con mayo considerado como periodo seco en la zona. Estos resultados sugieren que los periodos en los que se observaron los máximos picos poblacionales de $X$. volvulus son los que reunen las condiciones ambientales necesaria para el máximo desplazamiento mediante el vuelo y su establecimiento hacia su nuevo huésped, periodo en el que la alta humedad favorece el establecimiento de los hongos asociados de los cuales alimentaran a su progenie. En el caso de la Rec el pico poblacional que se presentó en abril puede deberse a que la zona de estudio es un área inundable donde aún en periodo de seca la humedad se mantiene alta, aunado a la vegetación de selva que tambien contribuye. Dentro de los factores ambientales que influyen en las poblaciones de insectos con hábitos que barrenan son la temperatura, lluvia, humedad, iluminación, disponibilidad de alimento, depredadores y competidores (Dajoz 2001). Las bajas poblaciones que se registraron en algunos sitios de estudio (El Bajío, Río Seco y REC) pueden deberse a las condiciones de manejo, la disponibilidad de recurso alimenticio, competencia y depredación que se presentan en las localidades, aunado a la edad de las plantas que componen los sitios de estudio, las cuales ejercen mayor resistencia al ataque de esta especie de insecto, al ser plantas jóvenes y vigorosas (Pérez et al. 2009).

AgRadecimientos. Los autores agradecen a los revisores por las aportaciones hechas al manuscrito, así como, a Ena Mata Zayas por las sugerencias al manuscrito y a los propietarios de las plantaciones de cacao que tan amablemente nos permitieron colectar en sus sitios de trabajo.

\section{LITERATURA CITADA}

Ascencio, R. J. M. \& Maldonado, M. F. 2002. El jardín Botánico Universitario "José Narciso Rovirosa" un esfuerzo para la conservación de la flora tabasqueña. Kuxulkab Revista de Divulgación, 7: 34-47.

Bustamante, O. F. \& Atkinson, T. H. 1984. Biología del barrenador de las ramas del peral Corthylus fuscus Blandford (Coleoptera: Scolytidae), en el norte del Estado de Morelos. Folia Entomológica Mexicana, 60: 83-101.

Cibrián, T. D., Méndez, M. J. T., Campos, B. R., Yates III, H. O. \& Flores, L. J. 1995. Insectos Forestales de México/Forest Insects of Mexico. Universidad Autónoma Chapingo. México. 453 pp.

Dajoz, R. 2001. Entomología forestal: los insectos y el bosque. Mundi-Prensa. Madrid. 548 pp.

INEGI (Instituto Nacional de Estadística Geografía e Informática). 2005. Cuaderno estadístico Municipal, Cárdenas, Tabasco, México. INEGI. México.187 pp.

INEGI (Instituto Nacional de Estadística Geografía e Informática). 2006. Cuaderno estadístico Municipal, Teapa, Tabasco. México. INEGI. México. 211 pp.

Iturre, M. \& Darchuck, E. 1996. Registro de escolítidos relacionados al género Eucalyptus en Santiago del Estero. Revista Quebracho, 4: 11-16. 
Morales, N. E., Zanuncio, J. C., Pratissoli, D. \& Fabres, A. S. 2000. Fluctuación poblacional de Scolytidae (Coleoptera) en zonas reforestadas con Eucalyptus grandis (Myrtaceae) en Minas Gerais, Brasil. Revista de Biología Tropical, 48: 101-107.

Palma-López, D. J., García L., E., Sol S., A. \& Juárez L., F. 1999. Plan de manejo de la Reserva Ecológica de la Chontalpa. Colegio de Postgraduados, Campus Tabasco y SEDESPA, Dirección de Ecología. H. Cárdenas, Tabasco, México. 77 pp.

Pérez, de la C. M., Equihua, M. A., Romero, N. J., Sánchez S., S. \& García, L.E. 2009. Diversidad, fluctuación poblacional y plantas huésped de escolítinos (Coleoptera: Curculionidae) asociados con el agroecosistema cacao en Tabasco, México. Revista Mexicana de Biodiversidad. 80: 779-791.

Wood, S. L. 1982. The Bark and Ambrosia Beetles of North and Central America (Coleoptera: Scolytidae), a Taxonomic Monograph. No. 6. Brigham Young University. Utah. 1327 pp.

Wood, S. L. 1986. A reclassification of the genera of Scolytidae (Coleoptera). Great Basin Naturalist Memoirs. 10:1-126. 